\title{
Impacto de la caries dental y enfermedad periodontal sobre la sexualidad de la mujer
}

\section{Impact of dental caries and periodontal disease on women's sexuality}

\author{
Fuentes Fernández $\mathrm{R}^{*}$, Oporto $\mathrm{V} \mathrm{GH} \mathrm{H}^{* *}$, Silva $M \mathrm{~A}^{* *}$, Soto $\mathrm{P} \mathrm{C}^{* *}$, Prieto $\mathrm{R}^{* * *}$, \\ Sanhueza $A^{* * * *}$, Cantín $M^{* *}$
}

\section{RESUMEN}

El desarrollo de la sexualidad es imprescindible para lograr un estado de salud integral en un individuo. El sistema estomatognático participa activamente en el desarrollo pleno y armónico de ésta, por lo que patologías como la caries dental y enfermedad periodontal, y la consecuente pérdida de dientes afectan el aspecto físico, psicológico y social de quien las padece. El propósito de esta investigación fue determinar si la caries dental, la enfermedad periodontal o pérdida de dientes afectan los rasgos afectivo, creativo y comunicacional en la sexualidad femenina.

La muestra se constituyó por 86 mujeres de entre 18 y 40 años, quienes presentaban caries dental, enfermedad periodontal o pérdida de dientes, a quienes se les realizó el examen oral confirmando el diagnóstico. A todo el grupo se aplicó una encuesta de 15 preguntas desarrolladas para este estudio.

Se observó que la presencia de signos y síntomas como halitosis, falta de dientes y alteraciones en la estética influyen en la sexualidad de la mujer, afecciones que aumentan al progresar la severidad de estas patologías. Dado el diseño y las condiciones del presente estudio, los resultados indican que la caries dental y enfermedad periodontal, así como sus consecuencias, tienen relación con las alteraciones en la sexualidad femenina, por lo que sería de gran importancia considerar estas patologías al momento de crear programas de salud bucal enfocándose en la salud integral de la mujer.

Palabras clave: Caries, enfermedad periodontal, sexualidad; salud oral.

\section{SUMMARY}

The development of sexuality is essential to achieve an integral health state in the individual. The stomatognathic system is actively involved in the full and harmonious development of the person, so diseases such as dental caries and periodontal disease and subsequent tooth loss affect the physical, psychological and social aspect of the patient. The purpose of this research was to determine whether dental caries, periodontal disease or tooth loss affect the emotional, creative and communicative traits of female sexuality.

The sample consisted of 86 women between 18 and 40 who had dental caries, periodontal disease or tooth loss, who underwent oral examination to confirm the diagnosis. A survey of 15 questions developed for this study was applied to the whole group.

It was observed that the presence of signs and symptoms such as halitosis, missing teeth and cosmetic alterations influence the sexuality of women, increasing with the progression of the severity of these pathologies.

* $\quad$ Centro de Investigación CIMOFIR, Facultad de Odontología. Universidad de La Frontera. Temuco, Chile. Departamento de Odontología Integral. Facultad de Odontología. Universidad de La Frontera, Temuco, Chile.

*** Departamento de Pediatría. Facultad de Medicina. Universidad de La Frontera, Temuco, Chile.

**** Departamento de Matemáticas y Estadística. Facultad de Ingeniería. Universidad de La Frontera. Temuco, Chile. 
Given the design and the conditions of this study, the results indicate that dental caries and periodontal disease and its consequences, are related to changes in female sexuality, so it would be very important to consider these pathologies to create oral health programs and to focus on the overall health of women.

Key words: Caries, periodontal disease, sexuality, oral health.

Fecha de recepción: 29 de octubre de 2014.

Aceptado para publicación: 20 de diciembre de 2014.

Fuentes Fernández R, Oporto V GH, Silva M A, Soto P C, Prieto R, Sanhueza A, Cantín M. Impacto de la caries dental y enfermedad periodontal sobre la sexualidad de la mujer. Av. Odontoestomatol 2015; 31 (4): 273-281.

\section{INTRODUCCIÓN}

La caries dental y la enfermedad periodontal son patologías altamente prevalentes que constituyen un problema de salud pública mundial. En Chile, el 100\% de la población de Nivel Socioeconómico (NSE) medio-bajo y bajo presenta caries, mientras que el 92,19\% de las personas entre 35 y 74 años presentan periodontitis (1). Ambas patologías pueden llevar a la pérdida dentaria comenzando en grupos jóvenes. En adultos de 35-44 años sólo un $20 \%$ conserva su dentadura completa, con un promedio de 6,5 dientes; disminuyendo a un $1 \%$ en adultos entre 65 y 74 años, con un promedio de 15,8 dientes perdidos (2).

Según el Ministerio de Salud de Chile (MINSAL), la salud oral afecta la calidad de vida de los individuos en términos de dolor, malestar, limitación y minusvalía social y funcional (3). Un $37 \%$ de la población mayor de 15 años declara que su salud oral afecta su calidad de vida siempre o casi siempre, mientras los mayores de 20 años se ven más afectados, probablemente, debido a que su deterioro bucal es mayor. La calidad de vida relacionada a la salud oral describe cómo ésta afecta las funciones de una persona, su salud psicológica y bienestar social. Esta asociación se hace más fuerte con el aumento de la edad, sobretodo en mujeres (4).

La pérdida de dientes supone una desvalorización de la autoestima y una disminución de la expresión facial en las personas, alterando la gesticulación y expresión de emociones, además de inducir un envejecimiento prematuro del rostro. Esto trae consecuen- cias, como juicios sociales y exclusión por los pares (5), junto a la disminución de la autoestima y autoimagen corporal. La apariencia de los dientes es más importante para las mujeres que para los hombres $(6,7)$, cuyo rol social está fuertemente asociado a la belleza corporal (8), donde sólo el $50 \%$ de la población está satisfecha con características como su sonrisa (9).

Según Fernández et al (10), la mutilación de cualquier parte del cuerpo, inclusive los dientes, genera una castración psicológica inconsciente que provoca una apreciación deficitaria del cuerpo, que por años se había considerado íntegro, generando un importante deterioro sobre la autoestima y la autoimagen. Esto se refleja negativamente en aspectos sexuales y de afectividad, reafirmando el papel del estado bucal en el adecuado bienestar social y psicológico (11).

El odontólogo, además de ser rehabilitador funcional, cumple un importante papel desde un punto de vista psicoemocional en el autoestima de las mujeres, ya que interviene directamente en la apariencia física de ellas, pudiendo prevenir e incluso solucionar daños en la salud psicológica y emocional, junto a rehabilitar la cavidad oral de las personas, que según la OMS, es parte integral y fundamental de la salud en general (12).

La sexualidad se describe como una dimensión esencial de las personas, una parte integral del ser humano y se asocia directamente con la percepción que el individuo tiene de sí mismo. Un desequilibrio en ella se manifiesta con problemas físicos, emocionales y de pareja (13). 
Si bien se ha reconocido la importancia de la salud oral, aún no se ha determinado si la caries, enfermedad periodontal y pérdida dentaria afectan de manera negativa aspectos psicológicos de la sexualidad femenina, diagnóstico que permitirá incluir estas necesidades en las políticas públicas de salud oral destinadas a la población de mujeres adultas de nuestro país. El objetivo de esta investigación fue determinar si la caries dental, la enfermedad periodontal o pérdida de dientes afecta los rasgos afectivo, creativo o comunicacional, en la sexualidad de la mujer.

\section{MÉTODOS}

Se desarrolló un estudio de corte transversal correlacional, incluyendo variables cuantitativas y cualitativas sobre las patologías de origen oral que afectan de manera negativa la sexualidad de las mujeres.

La muestra se constituyó de 86 mujeres, de entre 18 y 40 años de edad, quienes presentaban caries dental, enfermedad periodontal o pérdida de dientes, atendidas en la Clínica Odontológica Docente Asistencial (CODA) de la Universidad de La Frontera, en Temuco, Chile, por estudiantes que cursan el IV y V año de la Carrera de Odontología.

Los criterios que se tomaron en cuenta para la inclusión de las pacientes al cuestionario de la muestra fueron el nivel educacional, incluyendo a las que correspondieran, al menos, a Enseñanza Básica Completa, y también, la presencia de pérdida de dientes por caries o enfermedad periodontal. Los criterios de exclusión fueron embarazo, mujeres con alteraciones mentales, que sufrieran de alcoholismo, y quienes presentaron pérdida de dientes por indicación de ortodoncia o traumatismos dentoalveolares.

El tamaño muestral se obtuvo considerando un $95 \%$ de confianza, un $5 \%$ de error de muestreo y una prevalencia de caries dental y enfermedad periodontal de un $50 \%$.

Los datos fueron recolectados mediante una encuesta diseñada para esta investigación.

La encuesta consistió en catorce preguntas cerradas y una abierta, donde las encuestadas debieron elegir una respuesta según su percepción. La encuesta fue clasificada en cuatro ítems según los signos y síntomas descritos, en donde cada pregunta se relaciona con algún componente de la sexualidad de la siguiente manera: Las preguntas 1 a 3 corresponden al ítem Halitosis, las preguntas 4 a 9 corresponden al ítem falta de dientes, las preguntas 10 a 12 corresponden al ítem dolor y las preguntas 13 y 14 corresponden al ítem Impacto en la estética dental; la 15 corresponde a una pregunta abierta para responder según la experiencia personal (Tabla 1). Además, se consignó edad, previsión, nivel de estudios, diagnóstico periodontal e índice COPD (Caries Obturada Diente Pérdida) en cada caso.

En referencia a la enfermedad periodontal, los criterios que se consideraron para su identificación fueron los establecidos por el International Workshop for a Clasification of Periodontal Diseases and Conditions, organismo que establece como enfermedad periodontal al grupo de enfermedades multifactoriales que llevan a la destrucción del aparato de protección y de soporte del diente, clasificándola según su severidad en gingivitis y periodontitis (14). De esta manera, una vez que las pacientes terminaban la encuesta, se procedió a la revisión de las fichas clínicas de las mismas, para establecer la presencia o ausencia de enfermedad periodontal, además del resto de los criterios considerados.

Los datos se procesaron en una tabla Excel para estudiar la asociación entre las variables mediante el análisis descriptivo, prueba de chi cuadrado y $t$-test para dos muestras independientes.

Este estudio fue aprobado por el Comité de Ética de la Facultad de Medicina de la Universidad de La Frontera. Las participantes firmaron una carta de consentimiento informado para ingresar al estudio.

\section{RESULTADOS}

Se observaron porcentajes de respuestas positivas sobre el $50 \%$ en los cuatro ítems analizados, indicando alteración en la sexualidad por la presencia de estos signos y síntomas, positiva para halitosis en el $82 \%$, la estética en el $74 \%$, falta de dientes en el $65 \%$ y dolor en el $56 \%$. 


\section{TABLA 1.- ENCUESTA DE SEXUALIDAD}

\begin{tabular}{|c|c|c|}
\hline Preguntas & Sí & No \\
\hline $\begin{array}{l}\text { 1. ¿El mal aliento alguna vez se ha transformado en un impedimento para besar a su } \\
\text { pareja? }\end{array}$ & & \\
\hline $\begin{array}{l}\text { 2. ¿Debido al mal aliento mantiene una distancia para hablar ya sea con su pareja o } \\
\text { con otra persona? }\end{array}$ & & \\
\hline 3. ¿El mal aliento le da inseguridad al momento de seducir o intimar con su pareja? & & \\
\hline 4. ¿Ha tapado su boca al sonreír debido a la falta de dientes? & & \\
\hline 5. ¿Ha tapado su boca al hablar por su falta de dientes? & & \\
\hline 6. ¿Ha evitado besar a alguien por su falta de dientes? & & \\
\hline $\begin{array}{l}\text { 7. ¿La falta de dientes le da inseguridad al hablar o sonreír en frente a su pareja u otras } \\
\text { personas? }\end{array}$ & & \\
\hline $\begin{array}{l}\text { 8. ¿Siente que la falta de dientes la hace menos atractiva frente a su pareja u otras } \\
\text { personas? }\end{array}$ & & \\
\hline 9. ¿̇La falta de dientes le da inseguridad al momento de intimar con su pareja? & & \\
\hline 10. ¿El dolor de muelas la hace enojarse más o ser menos cariñosa con su pareja? & & \\
\hline 11. ¿El dolor de muelas ha disminuido sus ganas de intimar? & & \\
\hline 12. ¿El dolor de muelas, la ha hecho rechazar a su pareja al momento de intimar? & & \\
\hline $\begin{array}{l}\text { 13. ¿Siente que las manchas en sus dientes (caries, tapaduras, sarro) la hace menos } \\
\text { atractiva al momento de seducir a su pareja? }\end{array}$ & & \\
\hline $\begin{array}{l}\text { 14. ¿Ha sentido rechazo de su pareja debido a los problemas de su boca (caries, } \\
\text { manchas, falta de dientes, etc.)? }\end{array}$ & & \\
\hline $\begin{array}{l}\text { 15. ¿Que dificultades ha tenido con su pareja u otras personas debido los problemas de } \\
\text { su boca? }\end{array}$ & & \\
\hline
\end{tabular}

Las preguntas 1 a 3 corresponden al item Halitosis, las preguntas 4 a 9 corresponden al item falta de piezas dentarias, las preguntas 10 a 12 corresponden al item dolor y las preguntas 13 y 14 corresponden al item Impacto en la estética dental. La 15 corresponde a una pregunta abierta para responder según la experiencia personal.

En relación a la halitosis, el $82 \%$ de las mujeres afirmó que este signo influye en forma negativa en el componente afectivo de la sexualidad, mientras que el $77 \%$ afirmó que además influye en el rasgo comunicacional de la sexualidad. En cuanto a la falta de dientes, el $62 \%$ opinó que este signo influye en el aspecto afectivo, el $61 \%$ en el componente creativo y el $39 \%$ en el comunicacional. En relación con la estética, el $74 \%$ opinó que este parámetro afecta el aspecto creativo y sólo un $23 \%$ el afectivo. Finalmente, el 56\% afirmó que el síntoma de dolor influye negativamente en el componente afectivo de la sexualidad femenina.

Al evaluar el grado de severidad de la enfermedad periodontal en la sexualidad femenina, se estableció la prevalencia de patologías periodontales, observando Gingivitis (67\%), periodontitis leve (17\%), perio- dontitis moderada (10\%) y periodontitis severa (7\%). Al relacionar el ítem de preguntas de halitosis, el 73\% de las mujeres con gingivitis y el $100 \%$ de mujeres con periodontitis (leve, moderada y severa) afirman al menos en una respuesta que este signo afecta en algún ámbito su sexualidad. Estos resultados muestran una diferencia estadísticamente significativa $(\mathrm{p}=$ 0,0276 ). Desde el punto de vista de los componentes de la sexualidad, el $66 \%$ de las pacientes con gingivitis asevera una influencia negativa en el rasgo comunicacional y el $73 \%$ en el afectivo. En mujeres con periodontitis leve, moderada o severa, el $100 \%$ afirmó que la halitosis afectó los parámetros comunicacional y afectivo al menos en una pregunta del área.

Al relacionar el ítem de ausencia de dientes con enfermedad periodontal, se observa que las pacientes con diagnóstico de gingivitis presentan menor 
cantidad de respuestas afirmativas que indiquen alteración en la sexualidad por la falta de dientes (53,6\%), seguido de aquellas pacientes que padecen periodontitis leve, moderada y severa $(85,7 \%, 87,5 \%$ y $100 \%$ respectivamente, $\mathrm{p}<0,05)$, destacándose el $100 \%$ en los aspectos creativo y afectivo; y un $83 \%$ en el componente comunicacional (Figura 1).

Además, al analizar el efecto del número de dientes perdidos sobre la sexualidad, las mujeres que poseen un número mayor a 4 dientes perdidos, los porcentajes de respuestas que afirman algún problema en la sexualidad son mayores $(90 \%)$ a los valores de las mujeres con menos de 4 dientes perdidos $(43,2 \%)$ ( $p<0,0001)$. La falta de dientes se relaciona con los tres rasgos de la sexualidad estudiados.

En cuanto al número de caries y su relación con las afecciones en la sexualidad femenina, no se observó una asociación significativa ( $p>0,05)$.

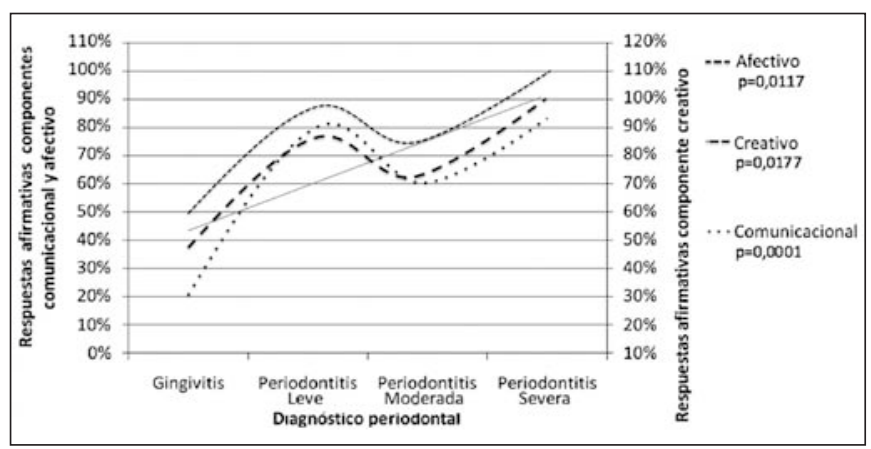

Fig. 1. Efecto de la falta de piezas dentarias en los componentes comunicacional, creativo y afectivo de la sexualidad, según la severidad de la enfermedad periodontal.
En el ítem de estética, se presentan diferencias significativas $(p<0,05)$, donde el $100 \%$ de las mujeres que presentan periodontitis severa o moderada, el $86 \%$ que presenta periodontitis leve y el $64 \%$ con gingivitis, afirmaron al menos en una pregunta que este signo afecta su sexualidad (Tabla 2). Por lo tanto, encontramos que, al aumentar la severidad periodontal, aumentaron las afecciones en los parámetros afectivo y creativo de la sexualidad $(\mathrm{p}<0,05)$.

Al correlacionar el dolor con la patología periodontal no se observaron alteraciones en relación a la sexualidad ( $p>0,05)$, demostrando que las alteraciones de la sexualidad por el síntoma dolor no se encuentran relacionado con el grado de severidad de la enfermedad periodontal. Sin embargo, se debe mencionar que todos los porcentajes superan el $50 \%$, lo que indica que el dolor, en forma independiente del grado de severidad periodontal que la paciente padezca, si afecta la sexualidad femenina.

Para analizar la influencia del COPD en la sexualidad femenina se calculó una mediana de 19, agrupando el índice dicotómicamente en menor o mayor/igual a $20(\mathrm{COPD}<20, \mathrm{n}=49$ y $\mathrm{COPD}=20, \mathrm{n}=35)$. En el grupo de mujeres con un índice COPD superior a 20 (Figura 2), los porcentajes de respuestas afirmativas en todos los ítems de preguntas estudiados fueron superiores a los valores obtenidos en las encuestas realizadas a las mujeres con índices de COPD menores a 20. Sin embargo, los porcentajes de respuestas afirmativas, de las mujeres con índice de COPD $<20$ fueron siempre sobre el $50 \%$, lo que demuestra que la historia de caries también

\section{TABLA 2.- EFECTO DE LOS ÍTEMS HALITOSIS, FALTA DE PIEZAS DENTARIAS, DOLOR Y ESTÉTICA SOBRE LA SEXUALIDAD DE LA MUJER, SEGÚN LA SEVERIDAD DE LA ENFERMEDAD PERIODONTAL}

\begin{tabular}{|l|c|c|c|c|c|}
\hline \multirow{2}{*}{ Ítems cuestionario } & \multirow{2}{*}{ Gingivitis } & \multicolumn{3}{|c|}{ Periodontitis } & P \\
\cline { 3 - 5 } & & \multicolumn{1}{|c|}{ leve } & moderada & severa & \\
\hline Halitosis & $73,2 \%$ & $100,0 \%$ & $100,0 \%$ & $100,0 \%$ & 0,0276 \\
Falta de piezas & $53,6 \%$ & $85,7 \%$ & $87,5 \%$ & $100,0 \%$ & 0,0121 \\
Dolor & $51,8 \%$ & $64,3 \%$ & $62,5 \%$ & $66,7 \%$ & 0,7512 \\
Estética & $64,3 \%$ & $85,7 \%$ & $100,0 \%$ & $100,0 \%$ & 0,0348 \\
\hline
\end{tabular}




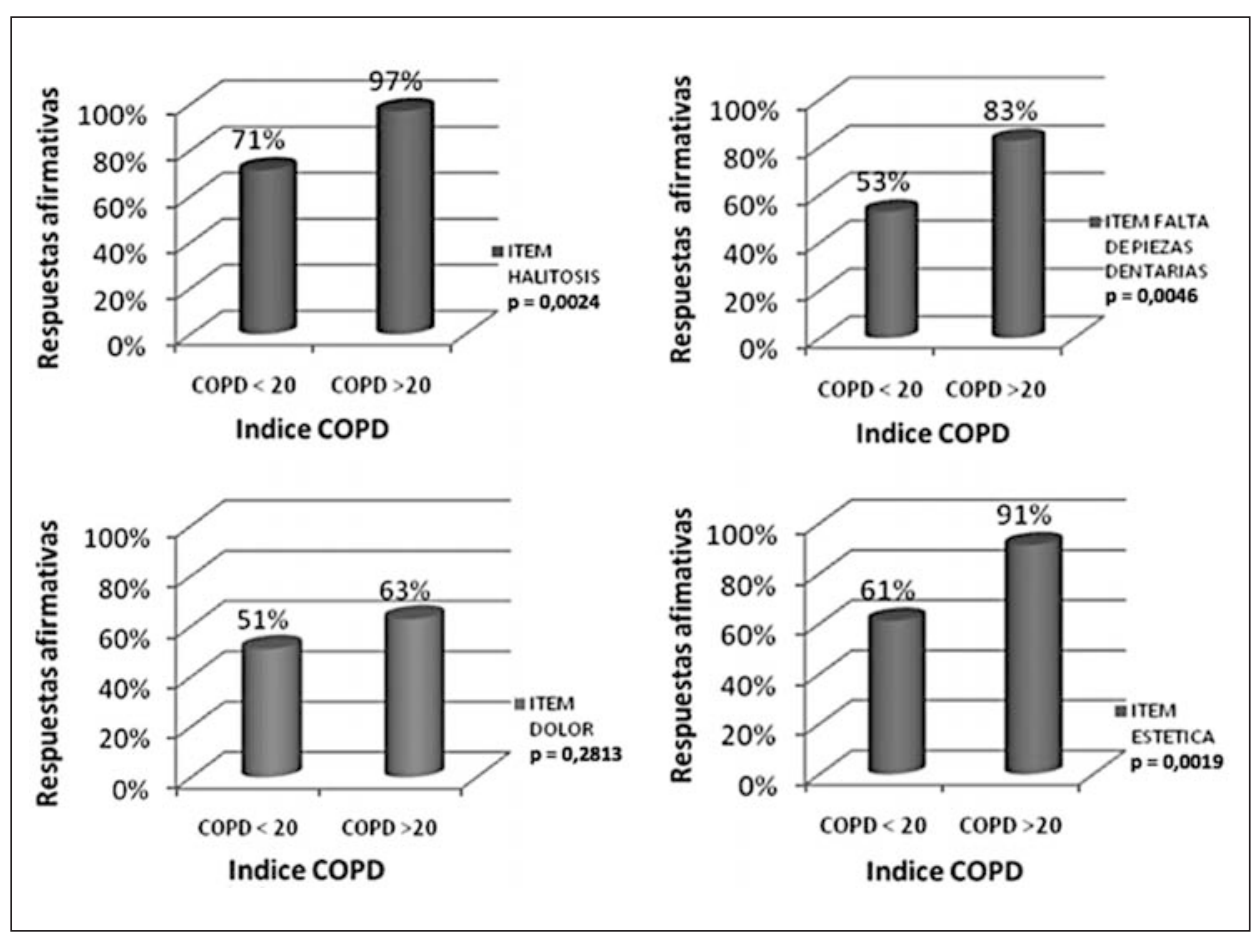

Fig. 2. Efecto de los signos y síntomas en la sexualidad de la mujer según valor del COPD. afecta la sexualidad a un porcentaje importante de mujeres.

En relación a la edad, las mujeres entre 36 y 40 años de edad presentan los porcentajes más altos de respuestas afirmativas que indican algún grado de alteración en la sexualidad por la presencia de los distintos signos y síntomas estudiados. Al asociar la previsión social con la sexualidad femenina no existe relación entre las variables. Los mismos resultados fueron observados al analizar el nivel de estudios con la sexualidad femenina.

Al analizar la pregunta abierta, se encontraron respuestas como:

- No tengo pareja porque me siento insegura.

- No he tenido pareja porque me da vergüenza no tener dientes.

- Siento vergüenza al hablar o sonreír, o

- El mal aliento me hace hablar de lejos con las personas.

En relación al autoestima, respondieron:

- Me siento fea y eso me baja la autoestima.

- Tengo baja autoestima.

- No quiero besar a mi pareja por mi mal aliento.

\section{DISCUSIÓN}

La sexualidad es un instinto básico, el cual presenta un conjunto de manifestaciones en el comportamiento humano, propias de la especie, influida por factores biológicos, psicológicos y socioculturales, donde un concepto integral de la sexualidad está presente durante toda la vida (13). En cada etapa existen necesidades y formas de expresar la sexualidad de manera diferente, involucrando diversos componentes. Patologías crónicas, como la enfermedad reumática, pueden afectar el funcionamiento sexual de varias maneras; los problemas físicos, emocionales y de pareja contribuyen a una vida sexual menos activa y menos disfrutable. El dolor crónico, la fatiga y una baja autoestima reducen el interés sexual y por ende la frecuencia del coito $(13,15)$. En el cáncer se reconoce un deterioro del autoestima y de la autoimagen en la mujer mastectomizada o histerectomizada, el cual se refleja negativamente en la posibilidad de ejercer una sexualidad normal (16). Sin embargo, la relación de la sexualidad y sus componentes con la caries dental y la enfermedad periodontal analizados en nuestra investigación, ha sido poco estudiada tanto a nivel nacional como mundial, no existiendo referencias anteriores. 
La mutilación de alguna parte del cuerpo, como ocurre con los dientes, es expresada verbalmente como la percepción de la pérdida de una parte erotizada del cuerpo, explicable como una castración psicológica que repercute en una apreciación disminuida de ese cuerpo que por años había sido considerado íntegro y ahora le da lástima (10).

La pérdida dentaria afectó al 65,5\% de las mujeres encuestadas, quienes opinaron que este signo afecta negativamente su sexualidad. Lambertini et al (6) consideran a los pacientes sin dientes como mutilados, a quienes les motiva a buscar atención dental para la preservación de una boca sana y una apariencia social aceptable. En nuestra investigación, en mujeres con más de 4 dientes perdidos, los porcentajes de respuestas en que se afirma que este signo afecta en algún aspecto la sexualidad aumenta a un $90 \%$, en comparación con aquellas mujeres con menos de 4 dientes perdidos, con un valor de 43,2\% $(p<0,0001)$. Patel et al (4) establecen que la calidad de vida tiene una relación directa con indicadores como número de dientes con movilidad, dientes perdidos, recesiones gingivales en zonas estéticas, así como otras afecciones periodontales, las que pueden evitar que los adultos expresen las emociones positivas que, a su vez, afectan su autoestima, así como sus interacciones sociales y personales.

Existe evidencia en relación a la estética dental donde sólo el $59 \%$ de las mujeres están satisfechas con la apariencia de su sonrisa (17). Para Samorodnitzky-Naveh et al (7) los principales factores que influyen en la satisfacción de la estética dental son las manchas en los dientes, el apiñamiento dentario anterior y las caries en dientes anteriores. Esto concuerda con lo expresado por las mujeres encuestadas en nuestra investigación, donde refieren que las alteraciones en la estética, provocadas por caries dental y enfermedad periodontal, afectan la sexualidad en un $73,8 \%$ de las pacientes. Además, al analizar la relación existente entre las alteraciones en la sexualidad versus la severidad de enfermedad periodontal, por presencia de alteraciones estéticas, se observó que los resultados demuestran que estas variables son directamente proporcionales, con valores significativos $(p<0,005)$, infiriendo que junto a una mayor severidad de la enfermedad periodontal aumentan los problemas en la sexualidad.
Esta tendencia se repite al valorar la influencia de la historia de la caries en la sexualidad femenina, donde con un mayor índice COPD, mayores fueron las alteraciones en la sexualidad por la presencia de este signo. Conjuntamente, el promedio del número de dientes perdidos fue estadísticamente mayor en aquellas mujeres que dicen sentir mayores problemas en su sexualidad.

La halitosis se observó como el signo que causa mayores alteraciones en la sexualidad femenina, en el $82,1 \%$ de los casos, intensificándose en mujeres mayores de 22 años, debido a que este grupo etáreo presenta una mayor prevalencia de este signo en comparación a las mujeres menores de 22 años.

Los hombres tienen tres veces más halitosis que las mujeres (18), sin embargo, la halitofobia afecta principalmente al sexo femenino en una relación 8:220, debido a la importancia que se le da al halito por parte de las mujeres. Por otra parte, nuestros resultados muestran que junto a una mayor severidad de la enfermedad periodontal y valores del índice COPD, mayores son las afecciones en la sexualidad femenina por la presencia de halitosis, con valores de $\mathrm{p}=$ 0,0276 y $p=0,0024$ respectivamente, demostrando la relación que existe entre las variables.

En cuanto al síntoma dolor, el 56\% de las mujeres encuestadas afirma que este signo afecta su sexualidad. A pesar de esto, al buscar la relación con el grado de severidad de enfermedad periodontal y caries dental, los valores encontrados no muestran diferencias estadísticamente significativas, por lo que no existe relación entre las variables. Sin embargo, es necesario destacar que las respuestas afirmativas superaron el $50 \%$, por lo que no se debe restar relevancia a este síntoma.

En relación a la edad, se observó una clara tendencia en el impacto de los signos y síntomas de las patologías nombradas al aumentar la edad, excepto en el dolor. Esto concuerda con lo reportado por López y Baelum (19), para quienes la presencia de enfermedad periodontal, pérdida de dientes y el aumento de la edad resultan tener un gran impacto en la calidad de vida de las mujeres. En las mujeres más jóvenes, de 18 a 22 años, sólo el $44 \%$ le dan una valoración a la estética dental, similar a lo repor- 
tado por Samorodnitzky-Naveh et al (7) en donde a los 21 años el 37,3\% estaba insatisfecho con su apariencia dental. Sin embargo, para Vallitu et al (20) la apariencia de los dientes es más importante en los pacientes más jóvenes.

Las patologías estudiadas afectan a la sexualidad femenina en forma transversal en relación al nivel de estudios y previsión de las mujeres analizadas.

Es indudable que signos y síntomas de las patologías bucales como la halitosis, la pérdida de dientes, la alteración de la estética y el dolor, afectan no sólo la apariencia, sino también el ámbito psicológico, factores como la autoimagen y las motivaciones $(17,21)$. Arango et al (22) afirman que una alteración en la autoimagen y el autoconcepto se manifiesta negativamente en la relación de la mujer consigo misma y con los demás, expresándose en el área sexual, donde a pesar de existir el deseo, éste se bloquea. Esto concuerda con los resultados obtenidos de nuestro estudio.

En conclusión, a partir de nuestro trabajo, creemos necesario considerar la salud bucal como un aspecto importante en la sexualidad femenina, para estimular la creación de programas de salud oral, que la conviertan en uno de los pilares para el desarrollo integral de la mujer dentro de nuestra sociedad, buscando la realización de nuevas investigaciones en el área.

\section{BIBLIOGRAFÍA}

1. Gamonal J, Mendoza C, Espinoza I et al. Clinical attachment loss in Chilean adult population: First Chilean National Dental Examination Survey. J Periodontol 2010;81:1403-10.

2. Ministerio de Salud de Chile. Evaluación Objetivos Sanitarios Salud Bucal 2000-2010. Disponible en: http://www.redsalud.gov.cl/portal/url/ page/minsalcl/g_proteccion/g_salud_bucal/ saludinmigrantespresentacion.html.

3. Ministerio de Salud de Chile. Informe Final Encuesta Calidad de Vida y Salud 2006, Julio 2007. Disponible en: http://epi.minsal.cl/epi/html/ sdesalud/calidaddevida2006/index.htm.
4. Patel RR, Richards PS, Inglehart MR. Periodontal health, quality of life, and smiling patterns -an exploration. J Periodontol 2008;79:224-31.

5. Van der Geld P, Oosterveld P, Van Heck G, Kuijpers-Jagtman AM. Smile Attractiveness Selfperception and Influence Personality. Angle Orthod 2007;77:759-65.

6. Lambertini A, Prosperi A, Guerra ME et al. Necesidades protésicas de los pacientes que acudieron al centro de atención a pacientes con enfermedades infectocontagiosas de la universidad Central de Venezuela entre noviembre del 2003 y noviembre del 2004. Acta Odontol Venez 2006; 44:342-5.

7. Samorodnitzky-Naveh GR, Geiger SB, Levin L. Patients' satisfaction with dental esthetics. J Am Dent Assoc 2007;138:805-8.

8. Díaz J, Blanquez MP. Corporalidad y Síntomas depresivos en adolescentes. Rev Psiquiatr Psicol Niño Adolesc 2001;1:16-25.

9. Jørnung J, Fardal $\varnothing$. Perceptions of patients' smiles: a comparison of patients' and dentists' opinions. J Am Dent Assoc 2007;138:1544-53.

10. Fernández SSMS, Ospina De González B, Múnera Garcés AM. La sexualidad en pacientes con cáncer de mama o cérvix sometidas a tratamiento quirúrgico en el Hospital General, Hospital San Vicente de Paúl e Instituto de Cancerología de la Clínica las Américas, Medellín, 1999. Rev Colomb Obstet Ginecol 2002;53:179-83.

11. González O, Solórzano A, Balda R. Estética en odontología: Parte I Aspectos psicológicos relacionados a la estética bucal. Acta Odontol Venez 1999;37:33-8.

12. Guzeldemir E, Toygar HU, Tasdelen B et al. Oral health-related quality of life and periodontal health status in patients undergoing hemodialysis. J Am Dent Assoc 2009;140:1283-93.

13. Xibillé-Friedmann D, Álvarez-Fuentes M, FloresFlores $G$ et al. Perception of sexuality in women 
with rheumatic disease: case-control pilot study. Reumatol Clin 2005;1:20-4.

14. Rioboo M, Bascones A. Factores de riesgo de la enfermedad periodontal: factores genéticos. Av Period 2005; 17:69-77.

15. Østensen M. New insights into sexual functioning and fertility in rheumatic diseases. Best Pract Res Clin Rheumatol 2004;18:219-32.

16. Zung WW. From art to science. The diagnosis and treatment of depression. Arch Gen Psychiatry 1973;29:328-37.

17. Akarslan ZZ, Sadik B, Erten H et al. Dental esthetic satisfaction, received and desired dental treatments for improvement of esthetics. Indian J Dent Res 2009;20:195-200.

18. Scully C, Greenman J. Halitosis (breath odor). Periodontol 2000 2008;48:66-75.

19. López R, Baelum V. Oral health impact of periodontal diseases in adolescents. J Dent Res 2007; 86:1105-9.
20. Vallittu PK, Vallittu AS, Lassila VP. Dental aesthetics -a survey of attitudes in different groups of patients. J Dent 1996;24:335-8.

21. Menéndez Collar M, Noguerol Rodríguez B, Cuesta Frechoso $S$ et al. Halitosis de origen periodontal: revisión. Av Periodon Implantol 2004; 16:19-33.

22. Arango AL, Botero B, Ossa LM. Algunos cambios en la calidad de vida manifestados por varias pacientes con cáncer de mama usuarias de la EPS. Medellín, Colombia: Seguro Social Medellín, Universidad Javeriana, Universidad de Antioquía; 1998;p. 40.

\section{CORRESPONDENCIA}

Prof. Dr. Ramón Fuentes Fernández

Centro de Investigación CIMOFIR

Facultad de Odontología

Universidad de La Frontera

Manuel Montt 112

Temuco, Chile.

Email: ramon.fuentes@ufrontera.cl 